Editorial

\title{
Acknowledgement to Reviewers of the International Journal of Financial Studies in 2014
}

International Journal of Financial Studies Editorial Office, MDPI AG, Klybeckstrasse 64, CH-4057 Basel, Switzerland

Published: 9 January 2015

The editors of the International Journal of Financial Studies would like to express their sincere gratitude to the following reviewers for assessing manuscripts in 2014:

\begin{tabular}{lll} 
Agliardi, Elettra & Gadinis, Stavros & Ma, Yue \\
Aguilera, Ruth V. & Gómez-Betancourt, Gonzalo & Marijon, Eloi \\
Alexander, Kern & Hájek, Petr & Maurer, Thomas \\
Ali, Hamid & Haque, Mahfuzul & Michael-Tsabari, Nava \\
Ambrose, Brent & Hill, James R. & Miletkov, Mihail \\
Arkes, Jeremy & Huang, Weihong & Mongelli, Francesco Paolo \\
Baldo, Mara Del & Humphreys, Brad & Nichols, Joseph B. \\
Benhmad, François & Ireland, Peter N. & Nikitopoulos Sklibosios, \\
Bergman, Michael & Jacobson, Bert H. & Christina \\
Bilgin, Mehmet Huseyin & Jaggi, Bikki & Obizhaeva, Anna \\
Borghesi, Richard & Jainaga, Txomin Iturralde & Phillips, Blake \\
Buckley, Ross & Kellermanns, Franz W. & Plyakha, Yuliya \\
Campopiano, Giovanna & Kiefer, Nicholas M. & Rohleder, Martin \\
Christopoulos, Apostolos G. & Kim, Kenneth A. & Safarian, Mher \\
Colli, Andrea & Kim, Young Sang & Schuermann, Til \\
Danuletiu, Dan Constantin & Knyphausen-Aufseß, Dodo zu & Seifried, Chad \\
Dekker, Julie C. & Kotlar, Josip & Shim, Jungwook \\
DiCicco-Bloom, Benjamin & Kowalewski, Oskar & Smith, Brian F. \\
Dontoh, Alex & Kurita, Takamitsu & Smith, Sarah \\
Dreger, Christian & Langley, Paul & Soebbing, Brian \\
Durán-Encalada, J.A. & Lin, Yaling & Surdej, Aleksander \\
Emmenegger, Susan & Liu, Chih-Liang & \\
\hline
\end{tabular}


Taylor, Karl B.

Watanabe, Nicholas
Wicker, Pamela

Wong, Clement
Wyrobek, Joanna M.

Xing, Haipeng

(C) 2015 by the authors; licensee MDPI, Basel, Switzerland. This article is an open access article distributed under the terms and conditions of the Creative Commons Attribution license (http://creativecommons.org/licenses/by/4.0/). 